\title{
Research on Ship Automatic Modeling and Identification System
}

\author{
Yao-jie Chen ${ }^{1,2,3, a)}$,Xin Yuan ${ }^{1,3, b)}$,Shu-lin Du ${ }^{1,3, c)}$, Can Song ${ }^{1,3, d)}$ \\ ${ }^{1}$ School of Computer Science and Technology, Wuhan University of Science and Technology, Wuhan \\ 430065, China. \\ ${ }^{2}$ School of Navigation, Wuhan University of Technology, Wuhan 430063, Hubei, China. \\ ${ }^{3}$ Hubei Province Key Laboratory of Intelligent Information Processing and Real-time Industrial System, \\ Wuhan 430065, China.

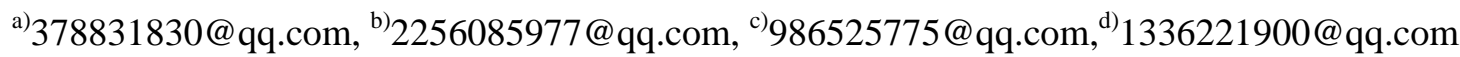

Keywords. automatic identification; six degrees of freedom; least square method

\begin{abstract}
When the ship sails in different water situation, it is necessary to forecast the movement state of the ship according to the real-time ship motion state parameters. The traditional method uses the classical MMG (mathematical model group) three-degree-of-freedom motion mathematical model to predict the modeling. For the existence of less detection parameters, the error is relatively large, the accuracy is not enough and so on. In this paper, a six-degree-of-freedom ship motion model based on sway, surge, heave, roll, pitch and yawing is proposed, combined with the least square method to achieve the automatic identification modeling method. Using this method, according to the GPS inertial navigation and positioning module to collect the data to simulate, and contrast with the traditional modeling method. The experimental results show that the improved automatic identification modeling method has better effect than the traditional modeling method, which greatly improves the accuracy of ship motion prediction.
\end{abstract}

\section{Introduction}

For the handling of the ship, generally only consider it in the horizontal plane movement. In actual circumstances, not only the ship moves in the horizontal plane after it at the helm, but also accompanied by rolling, pitching and first shake. But for most ships sailing, the range of roll, pitch and yawing is small, so the impact on the horizontal movement can be ignored, only study the ship in the horizontal movement. For the different water conditions and different hulls, when establish the accurate motion model, it's need to combine the least squares method and six-degree-of-freedom equation to identify the ship movement state.

This paper presents an automatic modeling method based on the six-degree-of-freedom ship motion model, which according to the classical MMG (Mathematical Model Group, separation mathematical model) three-degree-of-freedom ship plane motion coupled with roll, pitch and yawing, and combined with the least squares method. In this paper, a large cargo ship is used as the object of study. Based on the combination of least squares method and six-degree-of-freedom ship motion equation, the data collected from the cargo ship carrying GPS inertial navigation and positioning module are analyzed and use the data to deduce the automatic identification equation. And then the automatic identification equation is deduced by simulation analysis to verify its correctness. The method of automatic modeling identification provides an experimental reference model for the automatic positioning navigation of the ship and the automatic navigation. It provides an important experimental basis for the later ship navigation forecasting. 


\section{Six Degrees of Freedom Based on MMG}

The object has six degrees of freedom in space, that is, the degree of freedom of movement in the three orthogonal axes of $\mathrm{x}, \mathrm{y}$, and $\mathrm{z}$, and the degree of freedom of rotation about the three axes. Therefore, to fully determine the location of the object and movement transitions, we must determine the six degrees of freedom.

In this paper, two commonly used coordinate systems for ship maneuvering motion analysis are used: Inertial coordinate system and possessed coordinate system, see fig $1 . O_{0} x_{0} y_{0} z_{0}$ is an inertial coordinate system fixed to the surface of the earth, the $O_{0} \mathrm{x}_{0}$ axis points to the north, the $O_{0} \mathrm{y}_{0}$ axis points to the east, the $O_{0} z_{0}$ axis points to the center of the earth, the $O_{x y z}$ axis is the attachment coordinate system which the origin is in a designated point of the ship (for the simplified equation taken at the center of gravity), the $O_{x}$ axis pointing to the bow, the $O_{y}$ axis points to the starboard, the $O_{z}$ axis points to the bottom of the hull.

\section{Establishment of Ship 6 - DOF Motion Model based on MMG}

The actual movement of the ship is quite complicated, and in general there are six degrees of freedom. Surge Velocity $u$ 、Sway Velocity $v$ 、 Heave Velocity $w$ 、Yaw Rate $r$ 、 Rolling Rate $p$ 、 Pitching Rate $q$. In the inertial coordinate

system, the ship movement can use its three spaces position $X_{0}, Y_{0}, Z_{0}$ (or three space velocity $\left.\dot{X}_{0}, \dot{Y}_{0}, \dot{Z}_{0}\right)$ and three attitude angles which is the heading angle $\psi$ 、 the rolling angle $\varphi$ 、 Pitching Angle $\theta$ to describe, $(\psi, \varphi, \theta)$ is called Euler angle.

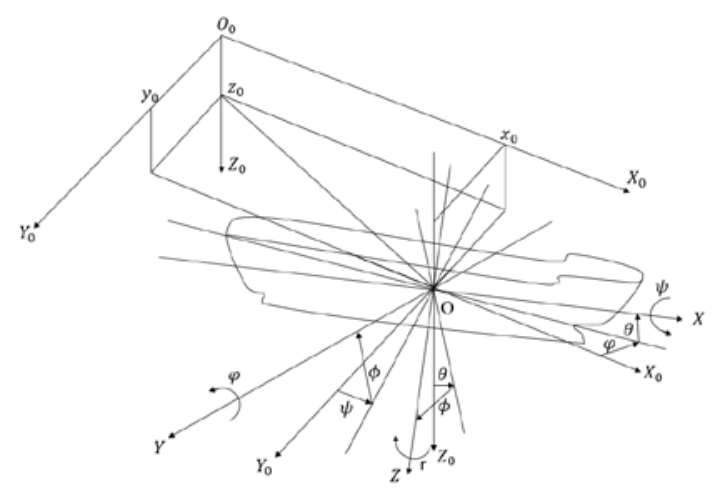

Figure 1 Ship motion coordinate system

According to the rigid body dynamics principle, the ship movement six degrees of freedom kinematic equation can be described as:

$$
\left\{\begin{array}{l}
m(\dot{u}-v r+w q)=X \\
m(\dot{v}-w p+u r)=Y \\
m(\dot{w}-u q+v p)=Z \\
I_{X} \dot{p}+\left(I_{Z}-I_{Y}\right) q r=K \\
I_{Y} \dot{q}+\left(I_{X}-I_{Z}\right) r p=M \\
I_{Z} \dot{r}+\left(I_{Y}-I_{X}\right) p q=N
\end{array}\right.
$$

In the equations, $m$ is ship quality; $I_{X} 、 I_{Y} 、 I_{Z}$ is the moment of inertia of the ship for the $G_{X}$ 、 $G_{Y} 、 G_{Z}$ axes,

$X 、 Y 、 Z$ and $K 、 M 、 N$ is the external force and the external moment acting on the hull. Assumptions:

(1) the ship sails in infinite wide waters;

(2) separate consideration of the forces of the ship, the propeller, the rudder and the waves, respectively;

(3) the impact of mutual interference between the ship, the paddle and the rudder;

(4) ignore the impact of the waves on the paddle and rudder, according to the basic idea of MMG separation modeling, will be applied to the hull on the external force and external force is 
divided into bare hull fluid power, propeller force, rudder force, wave force, can be said by equation (2) :

$$
\left\{\begin{array}{l}
\left(m+m_{x}\right)(\dot{u}+q w-r v)=X \\
\left(m+m_{y}\right)(\dot{\mathrm{v}}+r u-p w)=Y \\
\left(m+m_{z}\right)(\dot{\mathrm{w}}+p v-q u)=Z \\
\left(I_{x x}+J_{x x}\right) \dot{\mathrm{P}}+\left(I_{z z}-I_{y y}\right) \mathrm{qr}=K \\
\left(I_{y y}+J_{y y}\right) \dot{\mathrm{q}}+\left(I_{x x}-I_{z z}\right) \mathrm{pr}=M \\
\left(I_{z z}+J_{z z}\right) \dot{\mathrm{r}}+\left(I_{y y}-I_{x x}\right) \mathrm{pq}=N
\end{array}\right.
$$

In the equations:

$$
\begin{gathered}
X=X_{H}+X_{P}+X_{R}+X_{\text {wind }}+X_{\text {wave }}+X_{\text {current }} \\
Y=Y_{H}+Y_{R}+Y_{\text {wind }}+Y_{\text {wave }}+Y_{\text {current }} \\
Z=Z_{H}+Z_{\text {wind }}+Z_{\text {wave }} \\
K=K_{H}+K_{R}+K_{\text {wind }}+K_{\text {wave }}+K_{\text {current }} \\
M=M_{H}+M_{\text {wind }}+M_{\text {wave }} \\
N=N_{H}+N_{R}+N_{\text {wind }}+N_{\text {wave }}+N_{\text {current }}
\end{gathered}
$$

$X_{H} 、 Y_{H} 、 Z_{H} 、 K_{H} 、 M_{H}$ and $N_{H}$ are the force and moment of the bare hull in the corresponding six degrees of freedom; The variables with subscript $P$ and $R$ are the forces and moments of propeller and rudder respectively; The variables of wind 、 wave and current are the forces and moments of wind, wave and flow respectively; $m$ is ship quality; $m_{x} 、 m_{y} 、 m_{z}$ are the additional qualities of the longitudinal, transverse and vertical ships respectively; $I_{x x} 、 I_{y y} 、 I_{z z}$ are the moment of inertia around $O_{x} 、 O_{Y} 、 O_{z}$ axes respectively; $J_{x x} 、 J_{y y} 、 J_{z z}$ are the additional moment of inertia around $O_{X} 、 O_{Y} 、 O_{z}$ axes respectively; $u 、 V 、 W 、 p 、 q 、 r$ are the vertical, horizontal and vertical velocity and the angular velocity around $O_{x} 、 O_{Y} 、 O_{z}$ axis.

\section{Establishment of Ship Motion Model Based On Least Squares Identifications}

\section{The Establishment of Six Degrees of Freedom Nonlinear Model}

In order to simplify the mathematical equation that describe the motion of the ship, it can be considered that the center of mass of the hull ship is fixed. Then there is the following relationship between $p, q, r$ and $\varphi, \theta, \delta$ :

$$
\left[\begin{array}{c}
p \\
q \\
r
\end{array}\right]=\left[\begin{array}{ccc}
1 & 0 & -\sin \varphi \\
0 & \cos \varphi & \sin \varphi \cos \theta \\
0 & -\sin \varphi & \cos \varphi \sin \theta
\end{array}\right]\left[\begin{array}{c}
\dot{\varphi} \\
\dot{\theta} \\
\dot{\delta}
\end{array}\right]
$$

According to the rigid body dynamics theory, the movement of the hull around the centroid can be described by the mass moment of the mass system relative to the centroid of the mass, and the equations of the ship rotation are established:

$$
\left\{\begin{array}{l}
I_{x x} \dot{p}-I_{x x} \dot{r}+\left(I_{z z}-I_{y y}\right) q r-I_{x z} p q=K_{x} \\
I_{y y} \dot{q}+\left(I_{x x}-I_{z z}\right) p r+I_{x z}\left(p^{2}-r^{2}\right)=K_{y} \\
I_{z z} \dot{r}-I_{x z} \dot{p}+\left(I_{z z}-I_{y y}\right) q r-I_{x z} p q=K_{z}
\end{array}\right.
$$

In the equations, $I_{x x}, I_{y y}, I_{z z}, I_{x y}, I_{y z}, I_{x z}$ is the moment of inertia what take into account the additional mass effects. $K_{\mathrm{x}}, K_{\mathrm{y}}, K_{z}$ is the Hydrodynamic moment of the shaft ${ }^{x},{ }^{y},{ }^{z}$.

Using Newton's second law of motion, the equations of ship translation are established:

$$
\left[\begin{array}{ccc}
M+m_{x} & 0 & 0 \\
0 & M+m_{y} & 0 \\
0 & 0 & M+m_{z}
\end{array}\right]\left[\begin{array}{c}
\ddot{\varphi}_{c} \\
\ddot{\delta}_{c} \\
\ddot{\mu}_{c}
\end{array}\right]\left[\begin{array}{c}
F_{\varphi_{c}} \\
F_{\delta_{c}} \\
F_{\mu_{c}}
\end{array}\right]
$$

In the equations, $M$ is the quality of the hull; $m_{x}, m_{y}, m_{z}$ is the additional quality; $\ddot{\varphi}_{c}, \ddot{\delta}_{c}, \ddot{\mu}_{c}$ is the coordinates of the center of mass in the $E \varphi \delta \xi$ coordinate system; $F_{\varphi_{c}}, F_{\delta_{c}}, F_{\mu_{c}}$ is the component of hydrodynamic forces in the direction of the $E \varphi \delta \xi$ axis.

Combining the equation (4) and (5), we can get the six-degree-of-freedom nonlinear coupling equations of the ship's motion in the wave.

\section{Establishment of Nonlinear Model of Ship Horizontal}

It is generally believed that the ship's jerk movement has a significant effect on the roll motion 
and the first rocking motion, so the roll-first-roll coupling motion equation must contain the trending component. In the equation (3), make $\dot{\theta}=\theta=0$, unfolding the above triangular using Taylor formula and ignores the above four terms, $\sin x \approx x-x^{3} / 3 !, \cos x \approx 1-x^{2} / 2 !+x^{4} / 4$ !, the following formula holds:

$$
\left\{\begin{array} { l } 
{ p = \dot { \varphi } } \\
{ r = \operatorname { c o s } \varphi \cdot \dot { \xi } = ( 1 - \frac { \varphi ^ { 2 } } { 2 } ) \dot { \xi } = \dot { \xi } - \frac { \varphi ^ { 2 } } { 2 } \dot { \xi } }
\end{array} \text { and } \left\{\begin{array}{l}
p=\ddot{\varphi} \\
\dot{r}=\ddot{\xi}-\frac{\varphi^{2}}{2} \dot{\xi}-\varphi \dot{\varphi} \ddot{\varphi}
\end{array}\right.\right.
$$

Substituting equation (6) into equation (4) and making $q=\dot{q}=0$, get:

$$
\begin{aligned}
& \left\{\begin{array}{l}
I_{x x} \ddot{\varphi}-I_{x z}\left(\ddot{\xi}-\frac{\varphi^{2}}{2} \ddot{\xi}-\varphi \dot{\varphi} \ddot{\varphi}\right)=K_{x} \\
I_{z z}\left(\ddot{\xi}-\frac{\varphi^{2}}{2} \ddot{\xi}-\varphi \ddot{\varphi} \ddot{\varphi}\right)-I_{x x} \ddot{\varphi}=K_{z}
\end{array}\right. \\
& \left\{\begin{array}{l}
K_{x}=K_{\omega x} \cos \omega t+D_{x}+R_{x} \\
K_{z}=K_{\omega z}\left(\cos \omega t+\tau_{1}\right)+D_{z}+R_{z}
\end{array}\right.
\end{aligned}
$$

In the equation, $K_{\omega x}, K_{\omega z}$ is the amplitude of the roll and the first wave moment; $\omega$ is the frequency of encounter; $\tau_{1}$ is Phase difference; $D_{x}, R_{x}$ is the damping torque and the recovery torque of the roll, which can be approximate as:

$$
D_{x}=\mathrm{d}_{x} \dot{\varphi}, R_{x}=r_{x} \varphi+c_{x} \varphi \xi_{c}
$$

$D_{z}, \quad R_{z}$ is the first damping torque and recovery torque, which can be approximate as:

$$
D_{z}=\mathrm{d}_{z} \dot{\psi} \quad, \quad R_{z}=r_{z} \psi+c_{z} \psi \xi_{c}
$$

Substituting equation (5)、(6)、(7) into equation (4), get The Ship Roll - First - Shake Coupled Motion Equation:

$$
\left\{\begin{array}{l}
I_{x x} \ddot{\varphi}-I_{x x}\left(\ddot{\varphi}-\frac{\varphi^{2}}{2} \ddot{\psi}-\varphi \dot{\varphi} \ddot{\psi}\right) \\
=K_{w x} \cos \omega t+d_{x} \dot{\varphi}+r_{x} \varphi+c_{x} \varphi \xi_{c} \\
I_{z z}\left(\ddot{\psi}-\frac{\varphi^{2}}{2} \ddot{\psi}-\varphi \dot{\varphi} \ddot{\psi}\right)-I_{x z} \ddot{\varphi} \\
=K_{w z} \cos \left(\omega t+\tau_{1}\right)+d_{z} \dot{\psi}+r_{z} \psi+c_{z} \psi \xi_{c}
\end{array}\right.
$$

\section{Standard recursive least squares method}

The ship roll-first-decoupling motion system of equation (11) can be represented by the following model:

$$
y(t)=\varphi^{T}(t) \theta+\varepsilon(t)
$$

The principle of the least squares method is to find unknown quantities $\theta$ 's estimated value $\hat{\theta}(\mathrm{t})$, to minimize the sum of squares of model residuals:

$$
J=\sum_{\mathrm{t}=1}^{N} e^{2}(t)
$$

In the equation, $e(t)=y(t)-\varphi^{T}(t) \hat{\theta}(t)$. Making $Y(t)=[y(1), y(2), \cdots y(t)]^{T}, \quad \Phi(t)=[\varphi(1), \varphi(2), \cdots \varphi(t)]^{T}$, that the performance indicators $\mathrm{J}$ can be written:

$$
J=\left[Y(t)-\Phi^{T}(t) \hat{\delta}(t)\right]^{T}\left[Y(t)-\Phi^{T}(t) \hat{\delta}(t)\right]
$$

We can get the non-recursive least squares estimate based on the observation of $\delta$ to time t:

$$
\hat{\delta}(t)=\left[\Phi(t)^{T} \Phi(t)\right]^{-1} \Phi^{T}(t) Y(t)
$$

In order to eliminate the possible existence of the denominator zero, to avoid the matrix inversion of the operation, can be recursive gain least squares valuation:

$$
\begin{array}{r}
\hat{\delta}(t+1)=\hat{\delta}(t)+\frac{P(t) \varphi(t+1)\left[y(t+1)-\varphi^{T}(t+1) \hat{\delta}(t)\right]}{1+\varphi^{T}(t+1) P(t) \varphi(t+1)} \\
P(t+1)=P(t)+\frac{[P(t) \varphi(t+1)][P(t) \varphi(t+1)]^{T}}{1+\varphi^{T}(t+1) P(t) \varphi(t+1)}
\end{array}
$$

In the equation, $P(t)$ is covariance matrix, Initial value $\hat{\theta}(0)=0, P(0)=\gamma I \cdot \gamma$ is a very large positive number, and there are regulations: $r(t)=0, \delta(t)=0, t \leq 0$.

\section{Fast convergence iterative learning least squares method}


At time $t$, from the equation (12) can be obtained:

$$
y(t)=\varphi^{T}(t) \hat{\theta}(t)+e(t)
$$

After k iterations:

$$
y_{k}(t)=\varphi^{T}(t) \hat{\theta}_{k}(t)+e_{k}(t)
$$

Making:

$$
\begin{gathered}
e_{k}(t)=y_{k}(t)-\varphi^{T}(t) \hat{\theta}_{k}(t) \\
Y_{k}(t)=\left[y_{1}(t), y_{2}(t), \cdots, y_{k}(t)\right]^{T} \\
\Phi_{k}(t)=\left[\varphi_{1}(t), \varphi_{2}(t), \cdots, \varphi_{k}(t)\right]^{T} \\
\Omega_{k}(t)=\left[e_{1}(t), e_{2}(t), \cdots, e_{k}(t)\right]^{T}
\end{gathered}
$$

That can get:

$$
Y_{k}(t)=\Phi_{K}^{T}(t) \hat{\theta}_{k}(t)+\Omega_{k}(t)
$$

Consider the following indicator function:

$$
J_{k}\left(\hat{\theta}_{k}(t)\right)=\frac{1}{2}\left[Y_{k}(t)-\Phi_{K}^{T}(t) \hat{\theta}_{k}(t)\right]^{T}\left[Y_{k}(t)-\Phi_{K}^{T}(t) \hat{\theta}_{k}(t)\right]
$$

Similar to the recursive least squares method:

$$
\hat{\theta}^{L S}(t)=\hat{\theta}_{k-1}(t)+\frac{P_{k-1}(t) \varphi_{k}(t)}{1+\varphi_{k}^{T}(t) P_{k-1}(t) \varphi_{k}(t)} e_{k}(t)
$$

In the equation:

$$
P_{k}(t)=P_{k-1}(t)-\frac{P_{k-1}(t) \varphi_{k}^{T}(t) P_{k-1}^{T}(t)}{1+\varphi_{k}^{T}(t) P_{k-1}(t) \varphi_{k}(t)} e_{k}(t)
$$

The initial value is:

$$
e_{k}(t)=y_{k}(t)-\varphi^{T}(t) e_{k}(t)
$$

After $\mathrm{N}$ iterations, to the iteration termination condition, can get:

$$
\left\{\begin{array}{l}
P(t)=\mathrm{P}_{N}(t) \\
\hat{\theta}(t)=\hat{\theta}_{N}(t)
\end{array}\right.
$$

The general iteration termination condition is:

$$
\left\{\begin{array}{c}
\left\|\hat{\theta}_{k+1}(t)-\hat{\theta}_{k}(t)\right\|<\sigma \\
\text { else } \\
k=N_{\max }
\end{array}\right.
$$

In the equation: $\sigma$ is a small positive number, $N_{\max }$ is the setted maximum number of iterations.

In order to speed up the convergence rate, the P-type iteration is added. which is:

$$
\hat{\theta}_{k}(t)=\hat{\theta}^{L S} k(t)+\beta \varphi_{k}(t) \hat{e}_{k}(t)
$$

In the equation, $\beta$ is the P-type iteration coefficient, $e_{k}(t)=y_{k}(t)-\hat{\theta}_{k}^{\iota s}(t)$.

From the above, we can see that the basic steps of the fast convergence iterative learning least squares algorithm are as follows:

Step 1: For time t, according to the initial value $\mathrm{P}_{0}(t), \hat{\theta}_{0}(t)$ given by the equation (24), and set $k=0$.

Step 2: When the k-th run is repeated, update $\mathrm{P}_{k}(t)$ and $\hat{\theta}_{k}(t)$. match according to equation (23) and (27).

Step 3: According to equation (26) test iteration learning termination conditions, if the conditions are satisfied that turn step 4, otherwise $k=k+1$, turn Step 2.

Step 4: According to equation (24) to update $\hat{\theta}(t)$ and $P(t)$, $t_{0}$ turn step 1. 


\section{Experimental Data and Simulation}

\section{Experimental data}

The automatic modeling identification method requires data collected in the thousands of tonnage vessels sailing in the Three Gorges waters. Ship in the process of driving, high-precision inertial navigation module to collect the data, they are: vx,vy, $\mathrm{vz}$ (speed in the $\mathrm{x}, \mathrm{y}, \mathrm{z}$ axis in three directions of the component); $\mathrm{rx}, \mathrm{ry}, \mathrm{rz}($ angle in the $\mathrm{x}, \mathrm{y}, \mathrm{z}$ axis in three directions of the component); $\mathrm{ax}$ 、 ay 、 az(the acceleration is in the $\mathrm{x}, \mathrm{y}, \mathrm{z}$ axis components in three directions);wx,wy,wz(angular velocity in the $\mathrm{x}, \mathrm{y}, \mathrm{z}$ axis in three directions of the components). The data collected by the high-precision GPS positioning module, they are: v(ship movement speed), latitude and longitude. High-precision inertial navigation module sampling interval of $0.1 \mathrm{~s}$, and high-precision GPS positioning module sampling interval of $1 \mathrm{~s}$.

\section{Comparison of simulation tests}

In order to verify the accuracy of the ship's automatic modeling identification method, simulation testing is required. Experiments on three different methods of identification, Comparative Experiment Fitting system fitting results outputted Z systems outputting different identification parameter value of the model output and observed from the results obtained respectively with Figures 1, 2 and 3 description. Among them,curve 1 is the model output and curve 2 is the observed value.

\section{MMG ship motion prediction identification and manipulation of sports}

From the filtered Z-shaped test data, 400 samples are taken into the formula (1), and the values of $\mathrm{u}, \mathrm{v}, \mathrm{w}, \mathrm{p}, \mathrm{q}$ and $\mathrm{r}$ are obtained, and these parameters are substituted into the equation of motion to obtain the forecast result ,Figure1 :

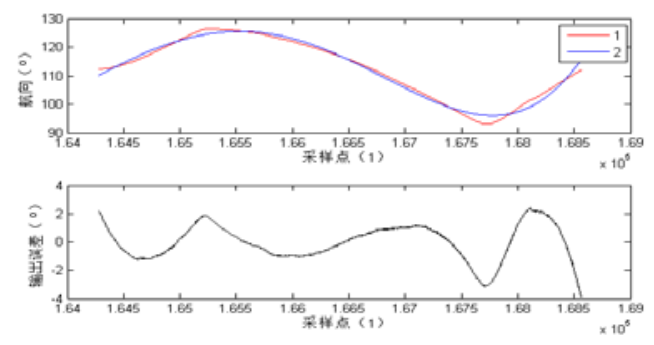

Figure 2 Z-type experimental system output fitting effect

The fitting error is 0.1191 and the correlation coefficient is 0.9858 .

\section{Standard recursive least squares identification and maneuvering motion prediction}

Taking the same value of the conventional motion modeling model samples, take the time $t_{0}$ to get the values of $\hat{\delta}(0) 、 \mathrm{P}(0)$, and successively substituted into equation (13) (14), after 400 iterations, obtained $\hat{\delta}(400) 、 \mathrm{P}(400)$ is substituted into the equation of motion prediction result obtained, as shown below.

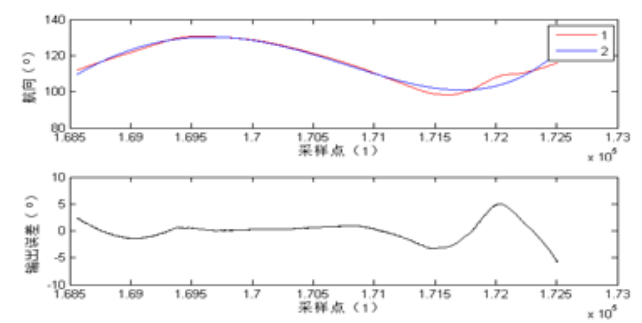

Figure 3 Z-type experimental system output fitting effect

The fitting error is 0.1087 and the correlation coefficient is 0.9890 . 


\section{Fast convergence iterative least squares identification and maneuvering motion prediction}

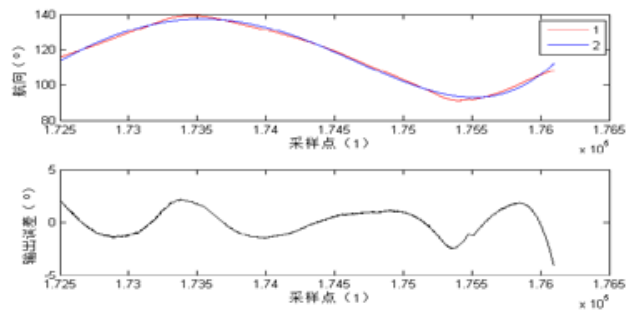

Figure 4 Z-type experimental system output fitting effect

From the modeling process can be obtained also calculated fitting error is 0.0962, the correlation coefficient is 0.9921

Analysis of Figure 1, Figure 2 and Figure 3 can be seen, the ship automatically modeling identification method to calculate the output and the results of the simulation curve is very close to the accuracy of the ship's automatic modeling method,and further improve the accuracy.

This method of automatic modeling and identification of the ship, through the specific experimental data collection and analysis and simulation, when the data reaches a certain number, the automatic modeling identification method to establish the model has to deal with most of the situation. According to the specific circumstances of the model has been established to repair, its operating timeliness and accuracy are very close to the actual situation of the data fitting. The automatic modeling method is based on the parameters of the ship 's six degrees of freedom and so on. The mathematical model is used to simulate the motion parameters of the ship in different situations.

\section{Conclusion}

According to the idea of MMG separation modeling, this paper establishes a large number of ship motion models by establishing the method of ship automatic modeling and identification, and predicts and simulates the ship motion state. In order to improve the accuracy of the motion model, based on the MMG identification of ship motion and the six - degree - of - freedom motion equation, the experimental results are compared with the standard recursive least squares method and the fast convergence iterative least squares method. The experimental results show that the MMG identification fit with the fast convergence iterative least squares ship motion has been significantly improved compared with the traditional identification. This method further improves the accuracy of ship motion prediction.

\section{Acknowledgments}

This work was financially supported by national science and technology support program (2015BAG20B05) and Hubei Provincial key laboratory of open fund (2016znss09B).

\section{References}

[1] YANG Peng. Research on Six-Degree-of- Freedom Motion Simulator and Its Control Method [D]. Harbin Engineering University,2008.

[2] HANG Xiu-Feng. Study on Mathematical Model of Six - Degree - of - Freedom Ship Motion in Navigation Simulator [D]. Dalian Maritime University,2009.

[3] SHI Chao. Study on Mathematical Model of Ship Maneuvering Simulation in Wind and Wave [D]. Harbin Engineering University,2011.

[4] JIA Xin-Le. Mathematical model of ship motion [D]. Journal of Dalian Maritime University

[5] MO Jian. Numerical Simulation of Six-Degree-of- Freedom Maneuvering Motion in Ship 
[D]. Harbin Engineering University,2009.

[6] YUE Mei-Long, LU Hui-Sheng, WANG Xi-Ling, NI Xue-Yi, CAI Cun-Qiang. Simulation Model of Ship Maneuvering Simulator [D]. Shanghai Jiao Tong University, Shanghai Maritime University,1998. 